\title{
On the Relationship Between the Practice of Mindfulness Meditation and Personality - an Exploratory Analysis of the Mediating Role of Mindfulness Skills
}

\author{
Paul A. M. van den Hurk • Tom Wingens • \\ Fabio Giommi • Henk P. Barendregt • \\ Anne E. M. Speckens • Hein T. van Schie
}

Published online: 18 June 2011

(C) The Author(s) 2011. This article is published with open access at Springerlink.com

\begin{abstract}
Mindfulness meditation (MM) has often been suggested to induce fundamental changes in the way events in life are experienced and dealt with, presumably leading to alterations in personality. However, the relationship between the practice of MM and personality has not been systematically studied. The aim of this study was to explore this relationship and to investigate the mediating role of mindfulness skills. Thirty-five experienced mindfulness meditators (age range, 31-75 years; meditation experience range, $0.25-35$ years; mean, $\sim 13$ years) and 35 age-, gender-, and ethnicity-matched controls (age range, 2763 years) without any meditation experience completed a personality (NEO-FFI) and mindfulness (KIMS) questionnaire. The practice of $\mathrm{MM}$ was positively related to openness and extraversion and negatively related to neuroticism and conscientiousness. Thus, the results of the current study associate the practice of MM with higher levels of curiosity and receptivity to new experiences and experience of positive affect and with less proneness toward
\end{abstract}

P. A. M. van den Hurk $(\bowtie) \cdot$ T. Wingens · F. Giommi •

H. P. Barendregt

Institute for Computing and Information Sciences,

Radboud University Nijmegen,

Heyendaalseweg 135,

6525AJ Nijmegen, the Netherlands

e-mail: p.vandenhurk@cs.ru.nl

\section{A. E. M. Speckens}

Department of Psychiatry,

Radboud University Nijmegen Medical Center,

PO box 9101, 6500HB Nijmegen, the Netherlands

H. T. van Schie

Behavioural Science Institute, Radboud University Nijmegen,

Montessorilaan 3,

$6525 \mathrm{HR}$ Nijmegen, the Netherlands negative emotions and worrying and a reduced focus on achievements. Furthermore, the mediating role of specific mindfulness skills in the relationship between the practice of MM and personality traits was shown.

Keywords Mindfulness meditation · Personality. Mindfulness skills $\cdot$ Mediation analyses

\section{Introduction}

In Eastern contemplative traditions, primarily Buddhism, the cultivation of mindfulness through the practice of meditation has a long history (Kabat-Zinn 1982; Linehan 1993). These traditions suggest that the regular practice of mindfulness meditation (MM) reduces suffering and fosters positive qualities, such as awareness, insight, wisdom, compassion, and equanimity (Baer et al. 2004; Goldstein 2002). Mindfulness can be described as paying attention in a special way, i.e., intentionally, in the present moment and nonjudgmentally (Kabat-Zinn 1996). In recent years, several questionnaires have been developed to operationalize the concept of mindfulness for scientific purposes (Baer et al. 2004, 2008; Brown and Ryan 2003; Walach et al. 2006). In these questionnaires, different mindfulness factors/skills have been defined, such as act-with-awareness (to fully engage in a current activity with undivided attention) and acceptwithout-judgment (to be non-evaluative about one's experiences in the present moment). Until now, several studies have investigated the relationship between these mindfulness skills and personality traits (Baer et al. 2004; Brown and Ryan 2003; Giluk 2009; Thompson and Waltz 2007). For example, in the study by Thompson and Waltz, mindfulness, as measured by the Mindful Attention Awareness Scale 
(Brown and Ryan 2003), was found to be positively related to agreeableness and conscientiousness and negatively related to neuroticism. The aim of these studies has been to examine to what extent mindfulness skills are related to personality traits. Remarkably, little or no attention has been directed at investigating whether and in what ways the practice of $\mathrm{MM}$ is related to personality traits. Attending this specific relationship seems to be important considering the fact that the practice of $\mathrm{MM}$ is aimed at inducing a fundamental change in the way events in life are experienced and dealt with (Kabat-Zinn 1990), presumably leading to alterations in personality.

Teasdale and colleagues have been among several other authors to give a meticulous description of the practice of MM (Bishop et al. 2004; Kabat-Zinn 1996; Ortner et al. 2007; Teasdale et al. 1995). They state: "In formal mindfulness practice, the student sits quietly in an erect and dignified posture and attempts, non-strivingly, to maintain attention on a particular focus, commonly his or her own breathing. When the attention wanders from the breath to the thoughts and feelings that inevitably arise, the student 'acknowledges and accepts' the thoughts or feelings, 'lets go' of them, and gently redirects attention back to the breath. This procedure is repeated many times, whenever the student notices that the attention has wandered. In informal practice, students apply the same general approach as often as possible during the course of their normal day, bringing the attention back to the 'here and now', using a focus on the breath as an 'anchor', whenever they notice that attention has been diverted to streams of thought, worries, reverie, or general lack of awareness" (Teasdale et al. 1995, p. 33). It has also been argued that MM promotes behavioral flexibility and that it fosters a decentered perspective from which subjective experiences are viewed as transient events rather than as permanent aspects of the self (Ortner et al. 2007).

Considering these descriptions of the practice of $\mathrm{MM}$, one can imagine the practice to have a large impact on one's mental state from moment to moment. For example, the clear intention to acknowledge and accept all thoughts and feelings as they arise in a non-judgmental way is in a sense revolutionary and can be hypothesized to reduce vulnerability to be lost in repetitive cycles of negative thoughts and worry. Accordingly, it can be expected that in the long term, one's personality is affected by the practice. In more detail, it seems plausible that the practice of MM is associated with reduced worry and negative thoughts, reflecting a reduced level of neuroticism. Likewise, the practice of MM, initiating voluntary exposure to a wide range of thoughts, emotions, and experiences, can be expected to be associated with increased levels in openness. Complementary to the prediction that the practice of MM is negatively related to neuroticism, MM can be expected to be positively related to extraversion, for extraversion stands for the experience of positive emotions and the tendency to be social, to engage with other people, among others. With respect to agreeableness, since agreeable people tend to be cooperative, supportive, caring, and concerned for others, the practice of MM, associated with feelings of empathy and compassion (Kabat-Zinn 1990), can be hypothesized to be positively related to agreeableness. Finally, since conscientious individuals are generally considered to be responsible, rule-abiding, and self-disciplined, the practice of MM can be expected to be positively associated with this personality trait (Giluk 2009).

The idea that the practice of MM might influence personality is contrary to traditional views which state that personality is a relatively stable construct that hardly changes across the life span (Heatherton and Weinberger 1994). However, recent studies advocate that change in personality may actually be possible (Helson et al. 2002; Piedmont 2001) and suggest that life experiences and environment play a significant role in personality development (Roberts et al. 2004). From this perspective, the practice of MM actually may be expected to lead to alterations in personality as $\mathrm{MM}$ is assumed to induce a significant shift in the way events in life are approached, experienced, and dealt with (Kabat-Zinn 1990).

In our view, mindfulness skills might play a crucial mediating role in the relationship between the practice of MM and personality traits. Mediation exists when one variable is indirectly related to another variable through at least one intervening variable, or mediator (Preacher and Hayes 2008). Accordingly, we hypothesize that the practice of MM might be indirectly related to personality traits, i.e., via mindfulness skills. For example, it seems plausible that the practice of $\mathrm{MM}$ is related to lower levels of neuroticism, as mentioned above. But it might very well be that this relationship exists due to the intervening mindfulness skill accept-without-judgment as this mindfulness skill is essential for the ability to acknowledge and accept (unwanted, negative) thoughts, feelings, and sensations in a nonjudgmental way and to let go of them, preventing one to get lost in repetitive cycles of negative thoughts and worrying. In our view, this change in perspective can provide a more complete and articulated view on the relationship between the practice of $\mathrm{MM}$, the cultivation of mindfulness skills, and personality traits.

Thus, the aim of this study was twofold: to investigate (1) the relationship between the (amount of) practice of MM and personality traits and (2) whether mindfulness skills have a mediating role in this relationship. On the basis of the descriptions of the MM practice given above and literature relating mindfulness facets to personality traits, we hypothesized the practice of MM to be positively related to openness, extraversion, agreeableness, and conscientiousness and 
negatively related to neuroticism (Giluk 2009). Mediation analyses were run for the personality traits that were shown to be related to the practice of MM. To explore the mediating role of mindfulness skills, four mindfulness skills (observe, describe, act-with-awareness, and accept-withoutjudgment), as defined by Baer et al. (2004), were considered as possible mediators.

\section{Methods}

Participants and Procedure

Thirty-five mindfulness meditators (mean (SD) age $=49.1$ (9.5)years, $11 \mathrm{men}$ ) and 35 age-, gender-, and ethnicitymatched control participants without meditation experience (mean (SD) age $=47.6$ (8.8)years, 12 men) participated in this study. Mindfulness meditators were recruited by sending invitation letters to MM centers in the Netherlands. Control participants were recruited by selecting participants from a participants database so that a control group was formed that matched the group of meditators on age $(t(68)=-0.66, p=$ $0.51)$, gender (Pearson $\left.\chi^{2}(1)=0.07, p=0.80\right)$, and ethnicity (all subjects were of the Caucasian race). We were not able to match the groups on education level (Pearson $\chi^{2}(2)=13.44$, $p<0.01$ ), and therefore education level was entered as a covariate in the statistical analyses. The mean amount of MM experience was 13.2 (SD 10.3)years, with a range of $0.25-35$ years in the mindfulness meditators group. Regular MM practice varied from 50 to 700 min a week, mean (SD) $3.0(2.2) \mathrm{h}$ a week. The control group had no experience with the practice of any form of meditation. None of the participants had any known psychological or neurological deficits. Participants completed the questionnaires before being tested in a cognitive experiment in which attentional processing was investigated. Before the start of the experiment, informed consent was obtained.

\section{Measures}

The NEO-Five Factor Inventory (NEO-FFI; Costa and McCrae 1992) was used to probe participants' scores on five personality factors: neuroticism (proneness toward negative emotions and worrying), extraversion (experiencing positive emotions and being sociable), openness-toexperience (having a receptive mind that attracts new experiences), agreeableness (the tendency to be altruistic, warm, and trustworthy), and conscientiousness (to be organized and have a high level of self-discipline). These personality factors were assessed with the authorized Dutch translation of the NEO-FFI (Hoekstra et al. 1996), a short version of the Revised NEO Personality Inventory (Costa and McCrae 1992). The NEO-FFI has 60 items, 12 per factor. Participants indicated their responses on a Likert-type scale ranging from 1 (strongly disagree) to 5 (strongly agree). Cronbach's alpha levels in the present study were 0.78 for neuroticism, 0.75 for extraversion, 0.72 for openness, 0.74 for agreeableness, and 0.81 for conscientiousness.

In addition to the measurement of personality, the scores on several mindfulness skills were probed by means of the Kentucky Inventory of Mindfulness Skills (KIMS; Baer et al. 2004). Four mindfulness facets are distinguished by this questionnaire: (1) mindful observation - the careful attending to and observing of internal and external phenomena (e.g., thoughts, emotions, smells, or sounds); (2) mindful description - the immediate, nonjudgmental labeling of observations, without conceptual analysis (Dimidjian and Linehan 2003); (3) act-withawareness - to fully engage in a current activity with undivided attention; (4) accept-without-judgment-to be non-evaluative about one's experiences in the present moment (see Table 1 for sample items). In order to probe the level of these mindfulness skills, participants filled in the validated Dutch version of the KIMS (Dekeyser et al. 2008). The KIMS consists of 39 items. Cronbach's alpha levels in the present study were 0.89 for observe, 0.89 for describe, 0.79 for act-with-awareness, and 0.85 for accept-without-judgment.

\section{Statistical Analyses}

First, by means of multivariate analysis (MANOVA; with group as between-subject factor, education level as covariate, and NEO-FFI factors as dependent variables), the scores of

Table 1 Sample items of the KIMS mindfulness factors observe, describe, act-with-awareness and accept-without-judgment

Observe

I notice changes in my body, such as whether my breathing slows down or speeds up.

I pay attention to how my emotions affect my thoughts and behavior. Describe

I'm good at finding the words to describe my feelings.

I can easily put my beliefs, opinions, and expectations into words.

Act-with-awareness

When I do things, my mind wanders off and I'm easily distracted. ${ }^{\text {a }}$

When I'm working on something, part of my mind is occupied with other topics, such as what I'll be doing later, or things I'd rather be doing. ${ }^{\text {a }}$

Accept-without-judgment

I criticize myself for having irrational or inappropriate emotions. ${ }^{\text {a }}$

I tell myself that I shouldn't be feeling the way I'm feeling. ${ }^{\text {a }}$

${ }^{\mathrm{a}}$ Reverse-scored item 
both groups on the NEO-FFI personality factors were compared. Second, in order to investigate whether the amount of MM experience (expressed in months) is related to personality traits, we restricted our correlation analysis to the group of meditators. Since personality traits have been found to vary across age (McCrae et al. 1999), we decided to control for this factor. In addition, we also controlled for education level and thus ran partial correlation analyses. Third, bootstrapping, a nonparametric resampling procedure, was used to test mediation (Preacher and Hayes 2008). Bootstrapping involves repeatedly sampling from the data set and estimating the indirect effect (i.e., the indirect pathway) in each resampled data set. By repeating this process thousands of times, an empirical approximation of the sampling distribution of the indirect pathway is built and used to construct confidence intervals for the indirect pathway.

\section{Results}

Prior to analyses, the data were examined for the presence of outliers. Therefore, each variable was standardized by computing the $z$ scores, and cases with a $z$ score higher than 2.58 or lower than -2.58 were defined as outliers. Four outliers were found (two per group) and were excluded. Thus, all analyses were performed on 33 participants in each group. Of note is that the mindfulness data of one meditator were missing; this participant was not included in the mediation analysis.

\section{The Practice of MM and Personality Traits}

The MANOVA on NEO-FFI personality factor scores was significant $\left(F(5,58)=3.52, p<0.01, \eta^{2}=0.23\right)$. Univariate analyses (with group as between-subject factor, education level as covariate, and a single NEO-FFI factor as the dependent variable) revealed a significant difference on openness-to-experience $\left(F(1,62)=10.42, p=<0.01, \eta^{2}=0.14\right)$, showing meditators to have a higher score on openness (see Table 1). In addition, meditators scored significantly lower on conscientiousness $\left(F(1,62)=4.04, p<0.05, \eta^{2}=0.06\right)$. No significant differences were observed for neuroticism, agreeableness, and extraversion ( $p>0.1$, Table 2).
Relationship Between Amount of MM Experience and Personality Traits

Partial correlation analyses showed MM experience (in months) to be related to neuroticism $(r=-0.39, p<0.05)$, extraversion $(r=0.36, p<0.05)$, and openness $(r=0.37, p<$ 0.05 ; see Table 3$)$. No significant relations were found between the amount of MM practice and either agreeableness or conscientiousness.

Mindfulness Skills as Mediating Factors in the Relationship Between the Practice of MM and Personality

Bootstrapping the indirect pathways in the relationship between the practice of $\mathrm{MM}$ and openness using 5,000 bootstrap samples (Preacher and Hayes 2008), describe (point estimate $(\mathrm{PE})=1.19,95 \%$ confidence interval $(\mathrm{CI})=0.20$ 3.21) and observe $(\mathrm{PE}=3.00, \mathrm{CI}=0.90-5.87)$ proved to be significant mediators. Describe $(\mathrm{PE}=2.24, \mathrm{CI}=0.48-4.54)$ and observe $(\mathrm{PE}=3.14, \mathrm{CI}=0.40-6.44)$ were shown to be mediators also in the relationship between MM practice and extraversion. In the relationship between MM practice and conscientiousness, describe $(\mathrm{PE}=1.96, \mathrm{CI}=0.49-4.38)$ and act-with-awareness $(\mathrm{PE}=1.02, \mathrm{CI}=0.10-2.77)$ were significant mediators. Finally, the mindfulness skills accept-withoutjudgment ( $\mathrm{PE}=-1.25, \mathrm{CI}=-3.09$ to -0.24$)$ and act-withawareness $(\mathrm{PE}=-0.90, \mathrm{CI}=-2.55$ to -0.02$)$ significantly mediated the relationship between the practice of MM and neuroticism (see Fig. 1). All the other investigated indirect pathways had the value of 0 included in the $95 \%$ confidence interval and were not considered significant mediating pathways.

\section{Discussion}

The objectives of the present study were twofold: first, to investigate the relationship between the practice of MM and personality traits and, second, whether mindfulness skills have a mediating role in this relationship. The findings of a higher level of openness in the group of meditators and the positive relationship between the amount of MM practice and openness were in line with earlier research as several studies
Table 2 Estimated marginal mean (SE) per group (meditators, non-meditators), univariate $F$ value, $p$ value, and $\eta^{2}$ for NEO-FFI personality traits

\begin{tabular}{lccrrr}
\hline NEO-FFI personality factor & Meditators $(N=33)$ & Non-meditators $(N=33)$ & $F$ & $p$ value & $\eta^{2}$ \\
\hline Neuroticism & $28.19(1.07)$ & $28.34(1.07)$ & 0.01 & 0.92 & 0.00 \\
Extraversion & $39.89(1.08)$ & $41.50(1.08)$ & 1.02 & 0.32 & 0.02 \\
Openness-to-experience & $43.91(1.05)$ & $38.91(1.05)$ & 10.42 & $<0.01$ & 0.14 \\
Agreeableness & $48.17(0.80)$ & $46.98(0.80)$ & 1.01 & 0.32 & 0.02 \\
Conscientiousness & $44.39(1.00)$ & $47.37(1.00)$ & 4.04 & $<0.05$ & 0.06 \\
\hline
\end{tabular}


Table 3 Partial correlations between MM experience and NEO-FFI personality traits (controlled for age and education level)

\begin{tabular}{lccccc}
\hline & Neuroticism & Extraversion & Openness & Agreeableness & Conscientiousness \\
\hline MM experience (months) & $-0.39^{*}$ & $0.36^{*}$ & $0.37^{*}$ & -0.09 & 0.02 \\
\hline
\end{tabular}

$* p<0.05$

showed that openness was positively related to facets of mindfulness (Baer et al. 2004, 2006; Brown and Ryan 2003). Thus, our results associate the practice of $\mathrm{MM}$ with higher levels in openness, a trait that is characterized by curiosity and receptivity to new experiences (Costa and McCrae 1992). At this point, it might be interesting to reflect upon the directionality of the relationship between the practice of MM and openness. On the one hand, it is possible that individuals scoring higher on openness might be more likely to start and continue with the practice of MM. However, on the other hand, the fact that the practice of MM initiates the voluntary exposure to a wide range of thoughts, emotions, and experiences suggests that increases in openness can be expected due to the practice of MM. Future longitudinal research is needed to definitely resolve this ambiguity.

The amount of MM experience was negatively related to neuroticism in the group of meditators, which is in line with several other studies that showed facets of mindfulness to be negatively related to neuroticism (Baer et al. 2004, 2006; Brown et al. 2007). It was surprising that no group difference on neuroticism was found in our study. This combination of results might be explained by the presence of baseline differences between the two groups, i.e., high neurotic people might be more likely to start with a MM practice, which in turn lowers the level of neuroticism over time. As such, a sample of expert meditators might become more akin to a non-meditator sample with respect to the level of neuroticism. It would be interesting for future research to

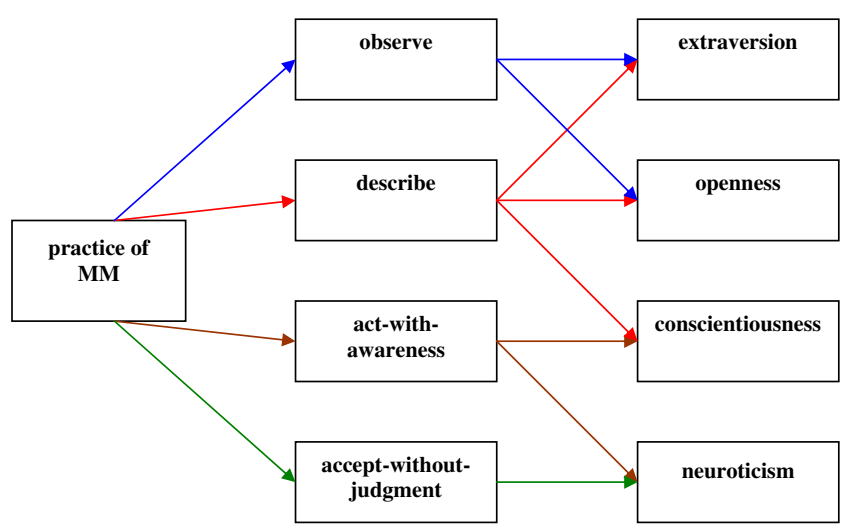

Fig. 1 Mediation of the relationship between the practice of MM and personality by the mindfulness skills observe, describe, act-withawareness, and accept-without-judgment investigate this hypothesis. In our view, these results are promising as they suggest that the practice of MM might help in reducing the experience of negative emotions.

The amount of MM experience was found to be positively related to extraversion, but no group difference was observed for extraversion. The positive relationship between the amount of MM experience and extraversion on the one hand, but the lack of a group difference on the other hand, seems to mirror the neuroticism results. This might actually be not so surprising. Whereas neuroticism reflects proneness toward negative emotions, extraversion reflects the experience of positive emotions. As such, high scores on neuroticism likely coincide with low scores on extraversion and vice versa. This inverse relatedness might explain the lack of group differences for both constructs and the reverse relationships of the constructs with the amount of MM experience. The positive relationship between the amount of $\mathrm{MM}$ practice and extraversion is in accordance with the results of, e.g., Brown and Ryan (2003) and Baer et al. (2004) and suggests that the practice of $\mathrm{MM}$ is associated with higher levels in the experience of positive affect.

Meditators scored significantly lower on conscientiousness than the control participants, but the amount of MM experience in the group of meditators was not related to conscientiousness. This pattern of findings suggests that people scoring low on conscientiousness might be more likely to start with the practice of MM. At first instance, the lower level of conscientiousness in the group of meditators seems somewhat puzzling since one hallmark of conscientiousness is self-discipline (Giluk 2009), a factor that is often considered essential for the continuity of the MM practice. Further consideration provided us with the idea that the level of conscientiousness might develop in a characteristic nonlinear way over the time of MM practice. In more detail, it seems plausible that people scoring low on conscientiousness - and so are little achievement-oriented (Barrick et al. 2001) might be more attracted to start with the practice of MM, given that the practice emphasizes not to focus on goals but rather on the process of reaching a goal. Then, in order to continue the practice, an increase in self-discipline might become essential, leading to higher scores on conscientiousness. However, over the time of practice, being mindful likely becomes a way of life rather than a, sometimes forced, formal practice for which a substantial amount of selfdiscipline is needed, reducing the level of conscientiousness 
again. This description might reconcile the results of Thompson and Waltz (2007) and our results. Namely, the positive relationship found in the study by Thompson and Waltz might be explained by the fact that only meditators with at most a couple of years of $\mathrm{MM}$ experience participated, reflecting the phase of increases in selfdiscipline. In our study, on the contrary, beginning, medium experienced, as well as expert meditators participated, so no linear relationship between the amount of MM practice and conscientiousness might be expected. Of note is that, albeit not significant, scores were indeed lower for the groups of beginning and expert meditators as compared with the group of medium experienced meditators in our study, supporting the idea of a nonlinear development (inverse U-shaped curve) of conscientiousness over the time of MM practice.

Meditators did not score higher on agreeableness than controls, and the amount of MM practice was not related to agreeableness in the group of meditators. These findings are in contrast with our predictions as well as with the results from the study of Thompson and Waltz (2007) who found a positive relationship between mindfulness skills and agreeableness. Furthermore, these results are not in line with the traditional view from Buddhism that the cultivation of mindfulness is related to greater compassion, for agreeableness reflects the characteristics of warmth and altruism. One possible explanation for the lack of a positive relationship between the practice of MM and agreeableness might be the lack of practice of metta meditation. During metta meditation, qualities such as compassion and loving kindness are cultivated, which are qualities that are very closely related to the concept of agreeableness. Although metta meditation is by some considered an indispensable element in the practice, the amount of time spent practicing metta meditation might vary a lot among individuals and traditions.

The mindfulness skill describe seems an important skill as it was shown to be one of the mediators of the relationship between the practice of MM and openness, extraversion, and conscientiousness. It has been suggested that the tendency to describe, label, or note experiences by covertly applying words leads to a greater ability to attend to new experiences in the present moment instead of being lost in repetitive cycles of thought and worry (Baer et al. 2004). Accordingly, the ability to describe can be hypothesized to result in a greater potential to self-regulate as opposed to react impulsively or habitually (Shapiro et al. 2006). In light of these descriptions, it makes sense why the mindfulness skill describe plays an important role in the relationship between the practice of MM and openness-to-experience, extraversion, and conscientiousness.

The mindfulness skill accept-without-judgment was shown to mediate the relationship between the practice of MM and neuroticism, which is a personality factor that is strongly and inversely related to psychological well-being.
There seems a clear ground for the idea that to acceptwithout-judgment plays an important role in the relationship between the practice of $\mathrm{MM}$ and neuroticism as to accept-without-judgment is to refrain from applying evaluative labels such as good/bad, right/wrong, or worthwhile/ worthless and to allow reality to be as it is without attempts to avoid, escape, or change it (Baer et al. 2004). Interestingly, in line with our results, in a recent study, the substantial negative relationship between mindfulness and anxiety and depression was completely due to the acceptance factor of the mindfulness construct (Kohls et al. 2009). Thus, the results of the current study seem to add evidence to the view that the development of the mindfulness skill accept-without-judgment is a key mechanism for improving psychological well-being. In addition, our findings extend this view to the healthy population by showing the important mediating role in a healthy population. It might be insightful for clinical studies as well as for prevention studies to focus on this factor.

One of the limitations of our study is the fact that our study was cross-sectional by nature, which places limits on the interpretation. Longitudinal research is needed to investigate whether a causal relationship exists between the practice of $\mathrm{MM}$, the resulting cultivation of mindfulness skills, and corresponding changes in personality. In addition, all of our data were self-reports, enabling the potential for single-source bias. It would be interesting for future research to investigate whether the pattern of results observed in our study is also obtained with the use of multiple other and externally verifiable sources such as family members and friends. We would also like to mention the fact that our proposed mediation models probably do not cover the complete relationship between the practice of MM, mindfulness skills, and personality traits. Part of the story also might be that people with certain personality traits are more likely to start a MM practice, which in turn leads to the cultivation of mindfulness skills. Nevertheless, the results of our study provided clear evidence to support the hypothesized relationship between the practice of $\mathrm{MM}$, mindfulness skills, and personality, as reflected by the plausible and specific mediating pathways that were found. Future research with meditation-naive participants who are at the beginning of their MM practice might be insightful regarding personality traits characteristic of people that are interested in meditation. Finally, the number of participants $(N=70)$ in our study might be considered relatively low, resulting in a possible power problem. Power analysis revealed that the power value was 0.89 for the MANOVA on NEO-FFI personality factor scores. This power value shows that the number of participants in our study was large enough to detect differences on NEO-FFI personality factors. 
The results of this study reveal a relationship between the practice of MM and personality traits. In more detail, the practice of MM was shown to be positively related to openness-to-experience and extraversion and negatively related to neuroticism and conscientiousness. Thus, the results of the current study associate the practice of MM with higher levels of curiosity and receptivity to new experiences and experience of positive affect and with less proneness toward negative emotions and worrying and a reduced focus on achievements. Furthermore, evidence was provided for the idea that specific mindfulness skills play a mediating role in the relationship between the practice of $\mathrm{MM}$ and personality traits.

Open Access This article is distributed under the terms of the Creative Commons Attribution Noncommercial License which permits any noncommercial use, distribution, and reproduction in any medium, provided the original author(s) and source are credited.

\section{References}

Baer, R. A., Smith, G. T., \& Allen, K. B. (2004). Assessment of mindfulness by self-report: The Kentucky inventory of mindfulness skills. Assessment, 11(3), 191-206.

Baer, R. A., Smith, G. T., Hopkins, J., Krietemeyer, J., \& Toney, L. (2006). Using self-report assessment methods to explore facets of mindfulness. Assessment, 13(1), 27-45.

Baer, R. A., Smith, G. T., Lykins, E., Button, D., Krietemeyer, J., Sauer, S., et al. (2008). Construct validity of the five facet mindfulness questionnaire in meditating and nonmeditating samples. Assessment, 15(3), 329-342.

Barrick, M. R., Mount, M. K., \& Judge, T. A. (2001). Personality and performance at the beginning of the new millennium: What do we know and where do we go next? International Journal of Selection and Assessment, 9, 9-30.

Bishop, S. R., Lau, M., Shapiro, S., Carlson, L., Anderson, N. D., Carmody, J., et al. (2004). Mindfulness: A proposed operational definition. Clinical Psychology: Science and Practice, 11, 230-241.

Brown, K. W., \& Ryan, R. M. (2003). The benefits of being present: Mindfulness and its role in psychological well-being. Journal of Personality and Social Psychology, 84, 822-848.

Brown, K. W., Ryan, R. M., \& Creswell, J. D. (2007). Mindfulness: Theoretical foundations and evidence for its salutary effects. Psychological Inquiry, 18, 211-237.

Costa, P. T., Jr., \& McCrae, R. R. (1992). Revised NEO Personality Inventory (NEO PI-R) and NEO Five-Factor Inventory (NEOFFI): Professional manual. Odessa, FL: Psychological Assessment Resources.

Dekeyser, M., Raes, F., Leijssen, M., Leysen, S., \& Dewulf, D. (2008). Mindfulness skills and interpersonal behaviour. Personality and Individual Differences, 44, 1235-1245.

Dimidjian, S., \& Linehan, M. M. (2003). Defining an agenda for future research on the clinical application of mindfulness practice. Clinical Psychology: Science and Practice, 10, 166-171.
Giluk, T. L. (2009). Mindfulness, Big Five personality, and affect: A meta-analysis. Personality and Individual Differences, 47(8), $805-811$

Goldstein, J. (2002). One Dharma: The emerging Western Buddhism. San Francisco: Harper Collins.

Heatherton, T. F., \& Weinberger, J. L. (1994). Can personality change? Washington: American Psychological Association.

Helson, R., Kwan, V. S. Y., John, O. P., \& Jones, C. (2002). The growing evidence for personality change in adulthood: Findings from research with personality inventories. Journal of Research in Personality, 36, 287-306.

Hoekstra, H. A., Ormel, J., \& de Fruyt, F. (1996). Handleiding NEO persoonlijkheidsvragenlijsten NEO-PI-R and NEO-FFI [Manual of the NEO personality inventories NEO-PI-R and NEO-FFI]. Lisse, the Netherlands: Swets Test Services.

Kabat-Zinn, J. (1982). An outpatient program in behavioral medicine for chronic pain patients based on the practice of mindfulness meditation: Theoretical considerations and preliminary results. General Hospital Psychiatry, 4, 33-47.

Kabat-Zinn, J. (1990). Full catastrophe living: The program of the Stress Reduction Clinic at the University of Massachusetts Medical Center. New York: Dell Publishing.

Kabat-Zinn, J. (1996). Mindfulness meditation: What it is, what it isn't, and its role in health care and medicine. In Y. Haruki, Y. Ishii, \& M. Suzuki (Eds.), Comparative and psychological study on meditation. Delft: Eburon.

Kohls, N., Sauer, S., \& Walach, H. (2009). Facets of mindfulnessResults of an online study investigating the Freiburg mindfulness inventory. Personality and Individual Differences, 46, 224-230.

Linehan, M. M. (1993). Cognitive-behavioral treatment of borderline personality disorder. New York: Guilford.

McCrae, R. R., Costa, P. T., Jr., de Lima, M. P., Simoes, A., Ostendorf, F., Angleitner, A., et al. (1999). Age differences in personality across the adult life span: Parallels in five cultures. Development Psychology, 35, 466-477.

Ortner, C. N. M., Kilner, S. J., \& Zelazo, P. D. (2007). Mindfulness meditation and reduced emotional interference on a cognitive task. Motivation and Emotion, 31, 271-283.

Piedmont, R. L. (2001). Cracking the plaster cast: Big Five personality change during intensive outpatient counseling. Journal of Research in Personality, 35, 500-520.

Preacher, K. J., \& Hayes, A. F. (2008). Asymptotic and resampling strategies for assessing and comparing indirect effects in multiple mediator models. Behavior Research Methods, 40(3), 879-891.

Roberts, B. W., Wood, D., \& Smith, J. L. (2004). Evaluating five factor theory and social investment perspectives on personality trait development. Journal of Research in Personality, 39, 166184.

Shapiro, S. L., Carlson, L. E., Astin, J. A., \& Freedman, B. (2006). Mechanisms of mindfulness. Journal of Clinical Psychology, 62 (3), 373-386.

Teasdale, J. D., Segal, Z., \& Williams, J. M. G. (1995). How does cognitive therapy prevent depressive relapse and why should attentional control (mindfulness) training help? Behavior Research and Therapy, 33, 25-39.

Thompson, B. L., \& Waltz, J. (2007). Everyday mindfulness and mindfulness meditation: Overlapping constructs or not? Personality and Individual Differences, 43, 1875-1885.

Walach, H., Buchheld, N., Buttenmuller, V., Kleinknecht, N., \& Schmidt, S. (2006). Measuring mindfulness - The Freiburg Mindfulness Inventory (FMI). Personality and Individual Differences, 40, $1543-1555$. 